\title{
Cohort study of Western Australia computed tomography utilisation patterns and their policy implications
}

\author{
David AJ Gibson ${ }^{1,2,3^{*}}$, Rachael E Moorin ${ }^{1,2}$ and C D'Arcy J Holman ${ }^{1}$
}

\begin{abstract}
Background: Computed tomography $(C T)$ scanning is a relatively high radiation dose diagnostic imaging modality with increasing concerns about radiation exposure burden at the population level in scientific literature. This study examined the epidemiology of adult CT utilisation in Western Australia (WA) in both the public hospital and private practice settings, and the policy implications.
\end{abstract}

Methods: Retrospective cohort design using aggregate adult CT data from WA public hospitals and Medical Benefits Schedule (MBS) (mid-2006 to mid-2012). CT scanning trends by sex, age, provider setting and anatomical areas were explored using crude $C T$ scanning rates, age-standardised $C T$ scanning rates and Poisson regression modelling.

Results: From mid-2006 to mid-2012 the WA adult CT scanning rate was 129 scans per 1,000 person-years (PY). Females were consistently scanned at a higher rate than males. Patients over 65 years presented the highest scanning rates (over 300 scans per 1,000 PY). Private practice accounted for $73 \%$ of adult CT scans, comprising the majority in every anatomical area. In the private setting females predominately held higher age-standardised CT scanning rates than males. This trend reversed in the public hospital setting. Patients over 85 years in the public hospital setting were the most likely age group $\mathrm{CT}$ scanned in nine of ten anatomical areas. Patients in the private practice setting aged $85+$ years were relatively less prominent across every anatomical area, and the least likely age group scanned in facial bones and multiple areas CT scans.

Conclusion: In comparison to the public hospital setting, the MBS subsidised private sector tended to service females and relatively younger patients with a more diverse range of anatomical areas, constituting the majority of CT scans performed in WA. Patient risk and subsequent burden is greater for females, lower ages and some anatomical areas. In the context of a national health system, Australia has various avenues to monitor radiation exposure levels, improve physician training and modify funding mechanisms to ensure individual and population medical radiation exposure is as low as reasonably achievable.

Keywords: Computed tomography, CT, Health service utilisation

\section{Background}

Computed tomography $(\mathrm{CT})$ scanning is considered to be a relatively high radiation dose diagnostic imaging modality [1]. In response to the growing utilisation of CT imaging [2,3], many nations have established or are in the process of implementing guidance and reference for the radiation dose delivered to the patient by $\mathrm{CT}$ examinations

\footnotetext{
* Correspondence: david.gibson@uwa.edu.au

${ }^{1}$ School of Population Health, University of Western Australia, Perth, Australia

${ }^{2}$ Faculty of Health Sciences, Curtin University, Perth, Australia

Full list of author information is available at the end of the article
}

[4-7]. The risk of cancer from CT scans and how best to incorporate these risks into clinical decisions are under review around the world [8-13]. However, discussion around funding mechanisms, access parameters, the consequent incentives and disincentives the organisation of health systems generate for undertaking these examinations has been lacking.

The increased availability and technological advancements of CT machines have resulted in growth and diversification of CT use well beyond the early days of in-patient head scans and cancer diagnosis [2,14-16]. A report from 
the United Nations Scientific Committee on the Effects of Atomic Radiation (UNSCEAR) showed CT represented only a few per cent of diagnostic procedures in developed nations, but was responsible for almost one half of diagnostic medical radiation exposure, and is the largest man-made source of ionising radiation exposure in the world [17]. The Organisation for Economic Co-operation and Development (OECD) has reported substantial increases in the per capita rate of CT scanning across the majority of developed countries [2]. The OECD noted increases in the per capita CT scanning rate of 34\% in France and 32\% in the USA between 2006 and 2010, while hospital CT scanning rates increased by $46 \%$ in the United Kingdom (UK) and 10\% in Canada between 2006 and 2010, and by 11\% in Ireland between 2009 and 2011 [2]. Interpretation of Australian CT scanning trends is complicated by the nation's mixed health funding arrangements; however, the OECD found an 18\% increase in nonpublic hospital CT scans from 2007 to 2011 [2] and reported Australia's non-public hospital CT scanning rate was 93.9 scans per 1,000 person-years (PY) compared with the OECD average of 131.8 scans per 1,000 PY in 2011 [18]. Other research has also noted an increasing utilisation of CT in Australia [3].

The bulk of Australia's non-public hospital CT (outpatient, private clinics and private hospitals) is subsidised by the federal government's Medicare Benefits Schedule (MBS), which also subsidises general practitioner (GP), pathology, other diagnostic, medical specialist and allied health services for all Australians on a fee-for-service basis. These are the CT services included within the OECD's estimates and are commonly performed at the request of a GP. CT scans performed within Australia's public hospitals are included within funding agreements established between the federal and state governments (who operate public hospitals) not under the MBS. The availability of public hospital CT data varies from state to state, leaving an incomplete picture of CT use in Australia.

CT Diagnostic Reference Levels (DRL) are the most common tool used by countries or large health systems to partially homogenise inter-practice radiation dose for the same examination by encouraging providers who exceed DRL parameters to reduce dose levels. However, DRL methodology relies on limited surveys of 10 to 20 'standard' CT scans on 'typical' patients by broad anatomical areas (e.g. Australian National DRL) or common clinical indications (UK DRL) voluntarily submitted by providers. Information collected during the DRL surveys does not include CT use epidemiology. Thus most national reports on $\mathrm{CT}$ radiation levels lack detail of who is being scanned (males or females, younger or older), the anatomical regions exposed and clinical indications for the scans [1]. With respect to guidelines for use of CT in Australia, there is no binding or regulatory scheme, although the Western Australian Department of Health has developed 'Diagnostic Imaging Pathways' guidelines to assist physicians in appropriate use of multiple radiological modalities [19].

When CT is used across the primary and tertiary care sectors as extensively as in Australia, universal DRLs lack context and risk irrelevance to either sector without differentiating their respective applications. Given the difference in the roles of public hospitals and the MBS subsidised services of the private and outpatient sector, it is reasonable to expect the profile of patients receiving CT scans would vary substantially across the two sectors. Subsequently the risk-benefit profiles of patients would also vary substantially when the interactions of machine output, patient sex, age, anatomical area and clinical indication(s) are taken into account [20]. These factors conspire to create unique patient risk profiles to be weighed against similarly unique potential benefits. Epidemiological profiles of system utilisation are essential to understand the differences between provider settings and the demands patients and clinicians are making of CT scans, but are currently lacking within the literature.

This study uses Western Australian (WA) CT utilisation data from the public hospital and MBS-supported settings to examine the epidemiology of adult CT scanning and explore the differences between the two service settings across sex, age and anatomical area to inform future policy options.

\section{Methods}

\section{Study design and data sources}

The study used a retrospective cohort design with three data sources to capture WA CT utilisation and population data from 1 July 2006 to 30 June 2012 (2006/07 to 2011/12 fiscal years):

- MBS billing data on adult CT scans provided aggregate counts of CT scans billed by out-of-hospital and private hospital providers [21]. The aggregate counts were categorised by sex, age group, year of claim and MBS item number. The data for patients aged 15 to 24 years were split by proportion to 18 to 24 years for comparability with public hospital data which was limited to patients 18 years and older.

- WA Department of Health public hospital adult CT scan data (inpatient, outpatient/clinic, emergency, private (Medicare), other/unknown) aggregated by year, gender, age group and scanning procedure code.

- Australian Bureau of Statistics (ABS) Census data provided population counts by gender, age and state each year [22]. 


\section{Rate of CT utilisation}

Crude rates of adult CT scans per 1,000 PY were calculated according to gender and age group in each year and for the entire study period (number of scans in population/number of people in population $\times 1000$ ). Population counts provided by the ABS provide the relevant population count at 30th June in each study year.

Age-standardised adult CT scan rates (per 1,000 PY) were calculated, using the direct method in STATA IC, according to gender, anatomical area scanned, and provider setting in each year for the study period.

\section{Adjusted CT utilisation likelihood}

Adjusted incidence rate ratios (IRR) of adult CT scans were estimated using Poisson regression (in STATA IC) following assessment of data for model appropriateness. Adult CT scans of each anatomical area within each provider setting were modelled separately by age group (patients age $85+$ years provided the reference group), adjusted for sex and year.

\section{Ethics}

This research was conducted with ethics approval from multiple ethics committees. Approval was given by the Government of Western Australia Department of Health Human Research Ethics Committee (project number \#2011/97), the Curtin University Human Research Ethics Committee (project number SMEC-80-10) and The University of Western Australia Human Research Ethics Committee (project number RA/4/1/1785). Informed consent was not required or collected as the data used in this study is collected routinely and aggregated.

\section{Results}

Public hospital CT scans could be performed under several 'admission' types: inpatient services were the most common (43\%), outpatient/clinic (30\%) and emergency patients $(26 \%)$ were the next most frequent; while only $1 \%$ of public hospital services were undertaken on private patients and thus billed directly to Medicare (data not shown). The MBS CT scans, with the exception of the small number performed by a public hospital, were all conducted in private radiological clinics, which may or may not have an affiliation with or proximity to a hospital (public or private).

\section{Rate of CT scanning in Western Australia}

Table 1 presents the number and crude rate of CT scans performed in WA from mid-2006 to mid-2012 by sex and age group in each financial year and over the total study period. The crude adult scan rate increased from 124.8 to $138.0 \mathrm{CT}$ scans per 1,000 PY, with an average of 128.8 CT scans per 1,000 PY for the study period.
Females were consistently scanned at a higher rate than males in each year. The 2011/12 female CT scan rate of 142.7 per 1,000 PY was $10 \%$ higher than the $2006 / 07$ rate. Males presented a similar trend, increasing from 119.4 CT scans per $1,000 \mathrm{PY}$ in $2006 / 07$ to $133.3 \mathrm{CT}$ scans per 1,000 PY in 2011/12.

A wide range of CT scanning rates was observed across age groups. A steep gradient from younger to the oldest adults was notable throughout the study period. The scanning rate of patients 18-24 years of age increased by $5 \%$ and the scanning rate of patients aged $25-44$ years increased by $12 \%$ from $2006 / 07$ to $2011 / 12$. The greatest variation over the study period occurred in the two oldest age groups, albeit with different patterns. The CT scanning rate in patients aged $65-84$ years increased by $18 \%$ to a $2011 / 12$ rate of 348.2 scans per 1,000 PY. However, the rate in those aged $85+$ years began higher at 400.6 scans per 1,000 PY and then declined by $11 \%$ over the study period.

\section{Division of CT services across provider settings}

Figure 1 presents the percentage of services performed in each provider setting for each anatomical area (including raw scan numbers) over the study period. The MBS setting provided at least $60 \%$ of all scans in each anatomical area, except for spiral angiography with 53\% of scans in the MBS setting. The anatomical areas most dominated by the MBS were facial bones (91\%) and spine (90\%). Head CT was substantially the most commonly scanned anatomical area in the public hospital setting despite only accounting for $40 \%$ of all head CT scans in WA.

Table 2 shows age-standardised adult CT scan rates for each anatomical area by sex, provider setting and year. Three anatomical areas presented consistently higher CT scan rates in the MBS sector for both males and females: head, abdomen/pelvis and spine. In the public hospital setting, two areas showed substantially higher scanning rates: head and abdomen/pelvis; however, the head scan rate was distinctly higher than the abdomen/pelvis rate. Most anatomical areas saw small increases in scanning rates in both provider settings; however some areas declined slightly in the MBS setting (head, neck, spine). The largest increase from 2006/07 to 2011/12 occurred in MBS intervention CT scans, rising from 7.3 to 11.4 scans per 1,000 PY.

In the MBS setting, females predominantly showed scanning rates higher than males, except for chest, spiral angiography, extremities and multiple areas CT scans. In the public hospital setting males presented with higher rates than females without exception.

Figure 2 presents the relative likelihood of a CT examination in each age group relative to patients aged $85+$ years (IRR $=1$ ) by anatomical area (modelled seperately) 
Table 1 Western Australia CT scan numbers and crude rates by sex and age group across the study period

\begin{tabular}{|c|c|c|c|c|c|c|c|c|c|c|c|c|c|c|}
\hline & \multicolumn{2}{|l|}{$2006 / 07$} & \multicolumn{2}{|l|}{$2007 / 08$} & \multicolumn{2}{|l|}{$2008 / 09$} & \multicolumn{2}{|l|}{$2009 / 10$} & \multicolumn{2}{|l|}{$2010 / 11$} & \multicolumn{2}{|l|}{$2011 / 12$} & \multicolumn{2}{|l|}{ Total } \\
\hline & $n$ & Rate* & $n$ & Rate* & $n$ & Rate* & $n$ & Rate* $^{*}$ & $n$ & Rate* & $n$ & Rate* $^{*}$ & $n$ & Rate* \\
\hline \multicolumn{15}{|l|}{ Sex } \\
\hline Female & 101,517 & 130.2 & 106,328 & 132.5 & 112,124 & 135.2 & 112,083 & 130.8 & 113,345 & 129.0 & 128,893 & 142.7 & 674,290 & 133.5 \\
\hline Male & 93,353 & 119.4 & 97,791 & 121.5 & 104,716 & 125.7 & 105,192 & 122.0 & 107,625 & 121.9 & 120,764 & 133.3 & 629,441 & 124.1 \\
\hline Total & 194,870 & 124.8 & 204,119 & 127.0 & 216,840 & 130.5 & 217,275 & 126.4 & 220,970 & 125.4 & 249,657 & 138.0 & $1,303,731$ & 128.8 \\
\hline \multicolumn{15}{|l|}{ Age group } \\
\hline $18-24$ years & 6,772 & 32.6 & 7,234 & 33.6 & 7,989 & 35.5 & 7,718 & 32.9 & 7,764 & 32.7 & 8,772 & 36.6 & 46,249 & 34.0 \\
\hline $25-44$ years & 39,724 & 66.7 & 40,817 & 66.8 & 43,018 & 68.1 & 42,003 & 64.1 & 41,360 & 61.5 & 48,438 & 69.9 & 255,360 & 66.2 \\
\hline 45-64 years & 74,026 & 143.5 & 77,160 & 145.3 & 81,120 & 148.0 & 82,029 & 145.6 & 82,687 & 143.2 & 92,803 & 156.9 & 489,825 & 147.2 \\
\hline $65-84$ years & 63,343 & 294.7 & 67,925 & 307.1 & 73,254 & 322.9 & 74,466 & 317.7 & 77,996 & 322.0 & 87,269 & 348.2 & 444,253 & 319.6 \\
\hline $85+$ years & 11,005 & 400.6 & 10,983 & 380.0 & 11,459 & 380.0 & 11,059 & 351.4 & 11,163 & 339.7 & 12,375 & 357.7 & 68,044 & 366.9 \\
\hline Total & 194,870 & 124.8 & 204,119 & 127.0 & 216,840 & 130.5 & 217,275 & 126.4 & 220,970 & 125.4 & 249,657 & 138.0 & $1,303,731$ & 128.8 \\
\hline \multicolumn{15}{|c|}{ Provider setting } \\
\hline MBS & 145,804 & 93.4 & 150,747 & 93.8 & 159,136 & 95.7 & 159,470 & 92.8 & 158,671 & 90.1 & 182,730 & 101.0 & 956,558 & 94.5 \\
\hline Public Hospital & 49,066 & 31.4 & 53,372 & 33.2 & 57,704 & 34.7 & 57,805 & 33.6 & 62,299 & 35.4 & 66,927 & 37.0 & 347,173 & 34.3 \\
\hline Total & 194,870 & 124.8 & 204,119 & 127.0 & 216,840 & 130.5 & 217,275 & 126.4 & 220,970 & 125.4 & 249,657 & 138.0 & $1,303,731$ & 128.8 \\
\hline
\end{tabular}

${ }^{*}$ Crude rate given in CT scans per 1,000 person-years.

in each provider setting. In the MBS setting, patients in age groups under 85 years were significantly more likely to undergo CT scanning of every anatomical area, except for head scans where there was no significant difference between the oldest two aged groups (Figure 2A). For the majority of anatomical areas in the MBS setting, patients aged $85+$ years had the second highest scan likelihood; except for CT of the facial bones and extremities where patients $85+$ years were least likely to be scanned. In the public hospital setting, patients aged $85+$ years were significantly more likely to have a CT scan than any other age group in all anatomical areas, except 'multiple areas' where patients aged 64-84 years were more likely (Figure 2B).

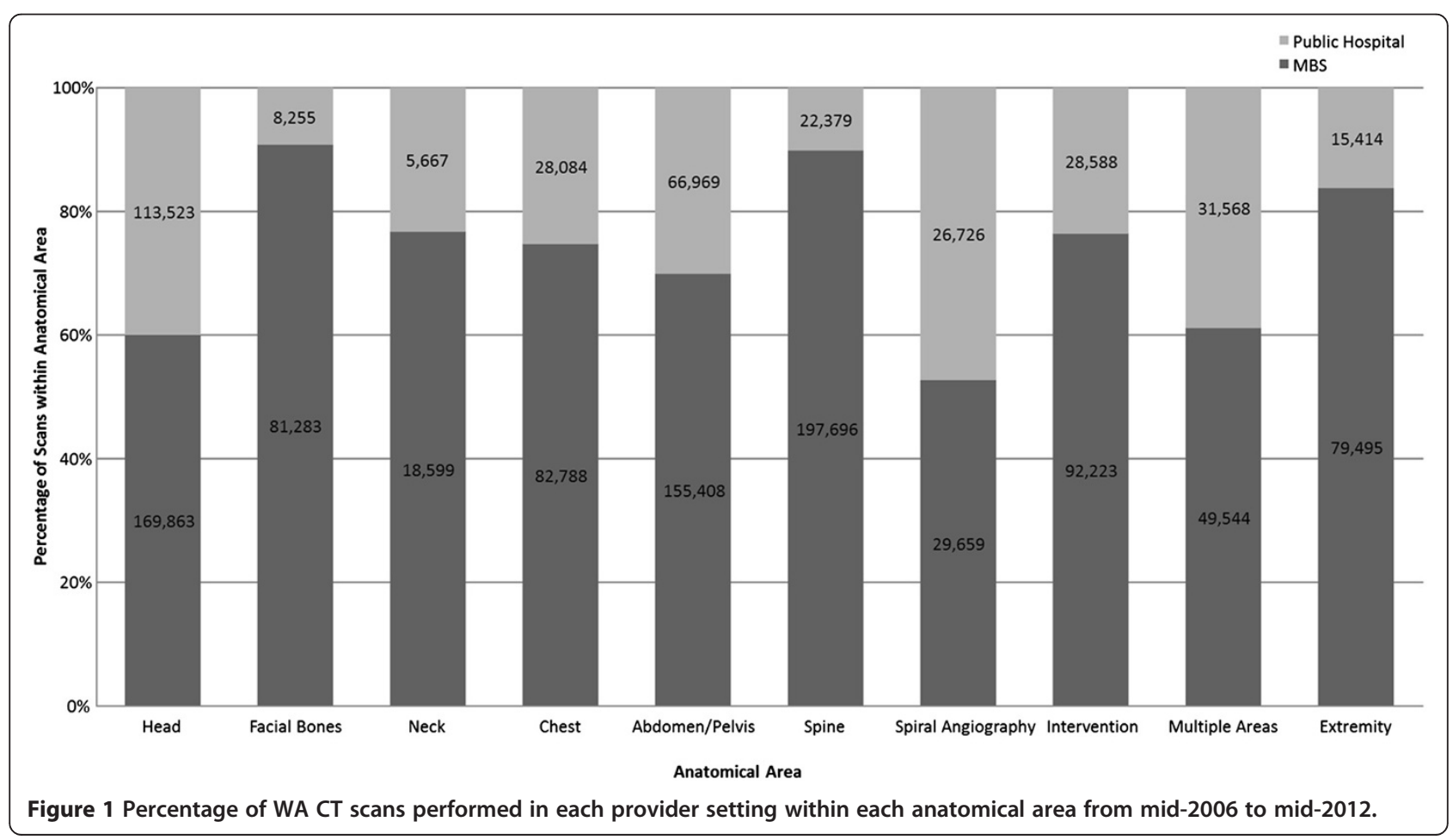


Table 2 Age standardised Western Australian CT scan rates (scans per 1,000 persons) by provider setting, anatomical area and sex

\begin{tabular}{|c|c|c|c|c|c|c|c|c|c|c|c|c|c|}
\hline \multirow[t]{2}{*}{ Sex } & \multirow[t]{2}{*}{ Anatomical Area } & \multicolumn{2}{|c|}{$2006 / 07$} & \multicolumn{2}{|c|}{$2007 / 08$} & \multicolumn{2}{|c|}{$2008 / 09$} & \multicolumn{2}{|c|}{$2009 / 10$} & \multicolumn{2}{|c|}{$2010 / 11$} & \multicolumn{2}{|c|}{$2011 / 12$} \\
\hline & & MBS & $\begin{array}{l}\text { Public } \\
\text { Hospital }\end{array}$ & MBS & $\begin{array}{l}\text { Public } \\
\text { Hospital }\end{array}$ & MBS & $\begin{array}{l}\text { Public } \\
\text { Hospital }\end{array}$ & MBS & $\begin{array}{l}\text { Public } \\
\text { Hospital }\end{array}$ & MBS & $\begin{array}{l}\text { Public } \\
\text { Hospital }\end{array}$ & MBS & $\begin{array}{l}\text { Public } \\
\text { Hospital }\end{array}$ \\
\hline \multirow[t]{10}{*}{ Male } & Head & 14.7 & 11.4 & 14.3 & 11.7 & 14.5 & 12.1 & 14.0 & 12.1 & 12.8 & 12.4 & 13.6 & 12.5 \\
\hline & Facial Bones & 6.9 & 0.9 & 6.7 & 1.0 & 6.4 & 1.1 & 6.1 & 1.0 & 5.8 & 1.0 & 8.3 & 1.0 \\
\hline & Neck & 1.5 & 0.7 & 1.7 & 0.6 & 1.8 & 0.7 & 1.7 & 0.6 & 1.6 & 0.7 & 1.8 & 0.7 \\
\hline & Chest & 8.6 & 3.3 & 8.3 & 3.4 & 8.7 & 3.2 & 8.2 & 3.0 & 8.6 & 3.3 & 8.9 & 3.4 \\
\hline & Abdomen/Pelvis & 13.4 & 6.5 & 13.9 & 7.0 & 14.4 & 7.3 & 13.8 & 7.1 & 14.0 & 7.7 & 15.0 & 8.5 \\
\hline & Spine & 18.8 & 2.3 & 18.5 & 2.6 & 18.8 & 2.8 & 17.4 & 2.6 & 15.5 & 2.7 & 17.1 & 2.8 \\
\hline & Spiral Angiography & 2.6 & 2.4 & 2.7 & 2.7 & 3.0 & 2.7 & 3.0 & 2.7 & 2.9 & 2.9 & 4.0 & 3.1 \\
\hline & Intervention & 5.6 & 3.2 & 6.2 & 3.4 & 6.7 & 3.5 & 7.7 & 3.4 & 8.6 & 3.5 & 9.7 & 3.1 \\
\hline & Multiple Areas & 3.8 & 3.4 & 4.2 & 3.2 & 4.8 & 3.4 & 5.1 & 3.4 & 5.3 & 3.5 & 6.0 & 3.6 \\
\hline & Extremity & 7.8 & 1.7 & 7.5 & 1.9 & 8.2 & 2.0 & 7.9 & 1.8 & 7.3 & 1.7 & 7.9 & 1.8 \\
\hline \multirow[t]{10}{*}{ Female } & Head & 21.0 & 9.6 & 20.8 & 9.6 & 20.4 & 10.6 & 19.4 & 10.3 & 17.8 & 10.9 & 18.6 & 11.2 \\
\hline & Facial Bones & 9.7 & 0.6 & 9.5 & 0.5 & 8.9 & 0.7 & 8.6 & 0.6 & 8.2 & 0.6 & 11.1 & 0.7 \\
\hline & Neck & 2.2 & 0.5 & 2.2 & 0.4 & 2.1 & 0.5 & 1.8 & 0.4 & 1.8 & 0.4 & 1.9 & 0.5 \\
\hline & Chest & 8.0 & 2.2 & 7.8 & 2.3 & 8.1 & 2.4 & 7.6 & 2.1 & 7.6 & 2.2 & 7.8 & 2.4 \\
\hline & Abdomen/Pelvis & 16.3 & 5.1 & 16.3 & 5.5 & 16.8 & 5.9 & 16.3 & 5.7 & 16.0 & 6.1 & 17.9 & 6.8 \\
\hline & Spine & 22.9 & 1.5 & 22.7 & 1.7 & 23.0 & 1.9 & 21.1 & 1.8 & 18.7 & 1.9 & 20.6 & 1.8 \\
\hline & Spiral Angiography & 2.2 & 2.2 & 2.5 & 2.5 & 2.7 & 2.3 & 2.8 & 2.4 & 2.8 & 2.7 & 3.8 & 2.9 \\
\hline & Intervention & 8.9 & 2.1 & 9.2 & 2.4 & 9.6 & 2.3 & 10.9 & 2.4 & 12.2 & 2.3 & 13.1 & 2.2 \\
\hline & Multiple Areas & 4.1 & 2.5 & 4.3 & 2.7 & 4.8 & 2.9 & 4.9 & 2.7 & 5.1 & 2.9 & 6.0 & 3.1 \\
\hline & Extremity & 7.5 & 1.2 & 8.0 & 1.3 & 8.2 & 1.2 & 7.9 & 1.2 & 7.6 & 1.1 & 8.5 & 1.3 \\
\hline \multirow[t]{10}{*}{ All } & Head & 17.9 & 10.5 & 17.6 & 10.7 & 17.4 & 11.3 & 16.7 & 11.2 & 15.3 & 11.6 & 16.1 & 11.9 \\
\hline & Facial Bones & 8.3 & 0.7 & 8.1 & 0.8 & 7.6 & 0.9 & 7.4 & 0.8 & 7.0 & 0.8 & 9.7 & 0.8 \\
\hline & Neck & 1.9 & 0.6 & 1.9 & 0.5 & 2.0 & 0.6 & 1.8 & 0.5 & 1.7 & 0.6 & 1.8 & 0.6 \\
\hline & Chest & 8.3 & 2.8 & 8.1 & 2.9 & 8.4 & 2.8 & 7.9 & 2.6 & 8.1 & 2.8 & 8.4 & 2.9 \\
\hline & Abdomen/Pelvis & 14.8 & 5.7 & 15.1 & 6.2 & 15.6 & 6.6 & 15.1 & 6.4 & 15.0 & 6.9 & 16.4 & 7.7 \\
\hline & Spine & 20.8 & 1.9 & 20.6 & 2.1 & 20.9 & 2.4 & 19.2 & 2.2 & 17.1 & 2.3 & 18.8 & 2.3 \\
\hline & Spiral Angiography & 2.4 & 2.3 & 2.6 & 2.6 & 2.9 & 2.5 & 2.9 & 2.6 & 2.9 & 2.8 & 3.9 & 3.0 \\
\hline & Intervention & 7.3 & 2.6 & 7.7 & 2.9 & 8.2 & 2.9 & 9.3 & 2.9 & 10.4 & 2.9 & 11.4 & 2.7 \\
\hline & Multiple Areas & 4.0 & 3.0 & 4.3 & 3.0 & 4.8 & 3.2 & 5.0 & 3.0 & 5.2 & 3.2 & 6.0 & 3.4 \\
\hline & Extremity & 7.7 & 1.4 & 7.7 & 1.6 & 8.2 & 1.6 & 7.9 & 1.5 & 7.4 & 1.4 & 8.2 & 1.6 \\
\hline
\end{tabular}

\section{Discussion}

In examining adult $\mathrm{CT}$ rates and the adjusted (i.e., independent) likelihood of receiving a CT scan in WA by provider setting, sex, age and anatomical areas across several years, our study provides a detailed picture of CT scan utilisation by adults in the Australian health care system. Importantly this study identifies differences in utilisation across the two provider settings previously assumed, but not quantified. Our study provides accurate estimates of adult CT utilisation rates in WA due to the coverage of both provider settings compared with previous estimates limited to only the MBS setting.
We observed an increase in CT scanning rates over six years across both provider settings in both sexes and in every age group except patients aged over 85 years. The most recent Australian CT scanning rates, published by the OECD is 2011 [2], were reported as 90.6 per 1,000 PY. In our study, the 2010/11 adult rate of CT utilisation was 125.4 per 1,000 PY. This is not surprising given that the OECD figures exclude the public hospital setting. However, a partial explanation could be differences in the range of ages covered by the rates, with this study restricted to the adult population aged 18 or more years. 


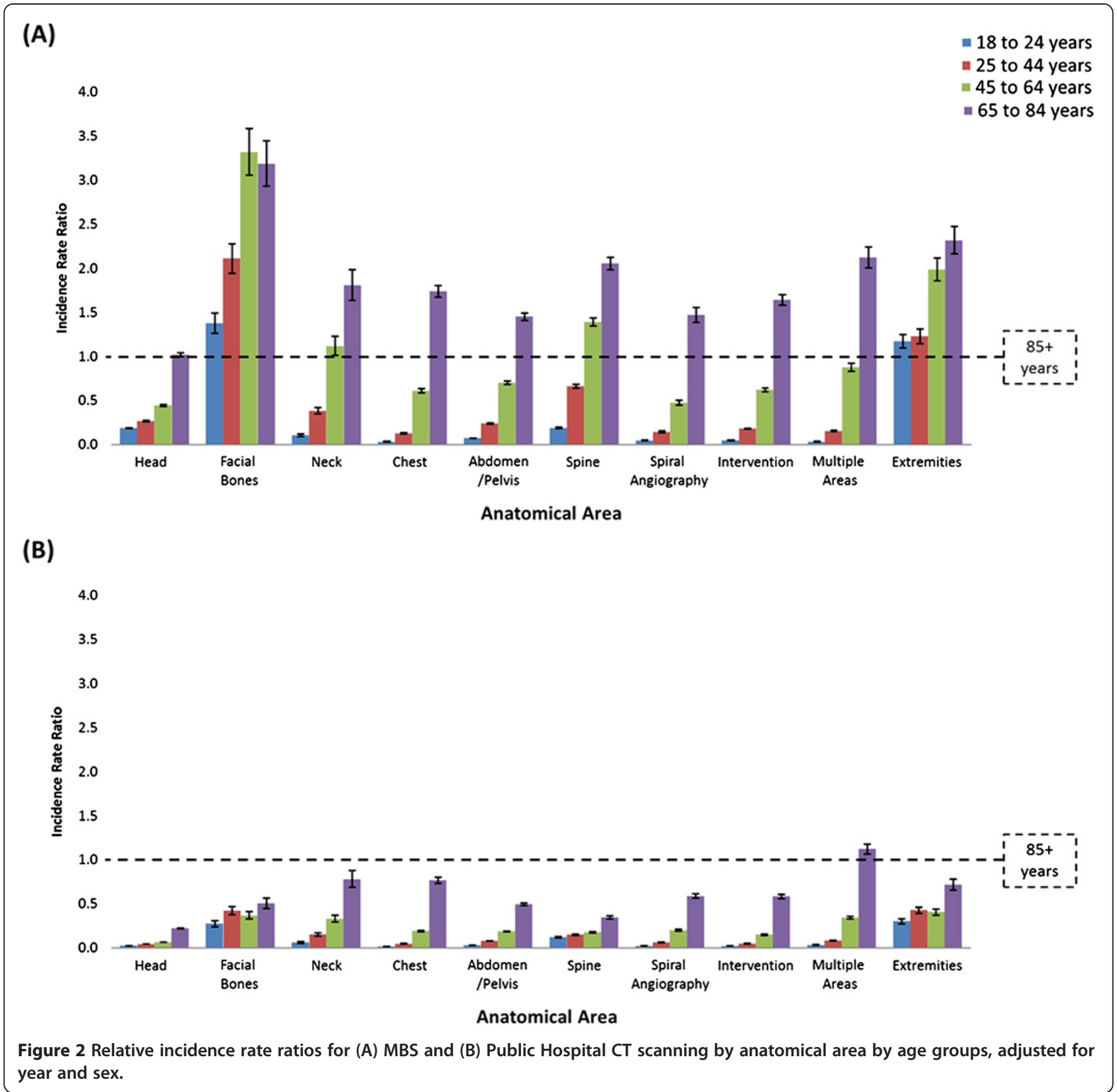

Our study showed substantial differences in the number, trends and typology of $\mathrm{CT}$ scanning undertaken in the two sectors. A larger number and rate of CT scans was undertaken in the MBS setting compared with public hospitals. In each year of the study the crude female CT scan rate was higher than in males, but deeper analysis revealed the sexes primarily utilise CT in different settings (females in the private practices and males in the public hospital setting). Patients aged over 85 years in the public hospital setting were the most likely age group to have a CT in nine of the ten anatomical sites. Conversely, younger age groups were more prominently represented in the MBS setting, especially patients 65 to
84 years of age. The starkest contrast between the two sectors occurred in scans of facial bones and extremities, where patients aged over 85 years were the least likely group to be scanned in the MBS setting, but the most likely group in the public hospital environment. Overall, the MBS setting supported a more diverse range of CT services to a younger, more female patient base in greater volume than the public hospital setting.

Evaluation of CT utilisation is crucial for several reasons. Radiation from CT scans delivers a population burden, as well as benefit, and this radiation cost-benefit is not equal across different groups of patients. Unnecessary (i.e., non-evidenced based) use of $\mathrm{CT}$ also places an 
unjustified cost on the health system. Thus review of service utilisation patterns can inform policymakers of the impact payment structures or competing modality uptake has on population burden and benefit. There are policy responses available within each of these domains to ensure efficacy, minimising risks and optimising cost effectiveness.

\section{Radiation risks for patients}

The linear-no-threshold model (i.e., no minimum safe level) is the prevailing theory of risk from radiation exposure [20]. As CT scanning develops new techniques, providing greater opportunity for utilisation, rigorous oversight and responsible access must reflect the principle of achieving radiation exposure that is as low as reasonably achievable (ALARA) $[1,23,24]$. The ALARA principle is primarily an application of common sense by medical personnel to: (a) minimise exposure time; (b) maximise distance from source; and (c) whenever possible, utilise shielding and barriers [1]. The ALARA principle is not only applicable at the patient level, but also at a systematic or population level.

Risks of radiation exposure to children are well publicised and inform a cautionary culture of radiation protection within paediatric services $[8,9,23]$. Radiation exposure risk is greater the younger an individual is at exposure [20]. However, while there is risk at exposure at all ages it does diminish, meaning when exposed to the same radiation middle-aged adults are at greater risk than older adults [20]. Additionally, females are at a greater risk from exposure than males at any age (not only due to cancers males are much less likely to develop, such as breast cancer) [20]. According the Committee on the Biological Effects of Ionizing Radiation's seventh report (BEIR-VII), the lifetime risk of all cancers for exposure to a single 0.1 Gray (Gy) radiation dose in females is 1.68 times higher than in males when exposed at age 20 years, 1.55 times higher if exposed at 30 years, 1.37 times higher at 40 years of age, 1.25 times higher at 50 years of age, 1.20 times higher at 60 and 70 years of age and 1.23 times higher at age 80 years [20]. Responsible rationing of high radiation imaging services delivers health system savings, not just in terms of utilisation rates and short-term expenditure; but also through a reduction in subsequent adverse outcomes such as radiation-induced cancer. Research from this team combining Australian MBS scanning records, collected $\mathrm{CT}$ dosimetry and the BEIR-VII exposure risk values identified a disproportionate burden risk of cancer incidence and mortality in females and younger adults [25].

Not only do the younger patients have greater time to develop cancer or are women more susceptible to ionising radiation burden, but particular anatomical sites carry differing radiosensitivities [17,26-28]. Application of the ALARA principle suggests the use of shields or barriers whenever possible. The prevalence of shield or barrier use in Australia by radiology services is unknown. Also of low prominence in discussions and research around medical radiation safety is the scan length of CT scans. While anatomical start and stop points will be used to guide scanning practices, machine over-scan and operator decisions can result in the inclusion of radiosensitive organs not intended for study or examination, such as the thyroid or liver during a chest scan that extends beyond the apices of the lungs or diaphragm. Inclusion of start and stop points for scans and the use of shields or barriers within industry guidelines or regulations could reduce unnecessary doses from certain scan types.

\section{Guidelines for medical radiation exposure}

In keeping with the ALARA principle, DRLs have been introduced in various countries to afford CT providers an indication of acceptable dosimetry values. Australia's own DRLs for adult CT, set by the Australian Radiation Protection and Nuclear Safety Agency (ARPANSA) [29], are relatively new compared with the UK and European Union guidelines $[28,30]$. Participation of practices in submitting data to DRL surveys and audits of practice performance against the DRLs are undertaken at the discretion of each provider in most nations including Australia [28-30]. Previous research in WA revealed substantial variation in CT scanning protocol settings and subsequent estimated radiation doses across provider settings [7]. Using a DRL survey methodology across a small sample of providers in WA for a selection of common CT scanning protocols, it was found these WA providers used higher dose scans than reported internationally for every scanning scenario [7].

With expanded technological capacities of CT machines and database systems, such as Picture Archiving and Communication Systems and Digital Imaging and Communications in Medicine fields, automatic collection of CT machine settings and dose outputs is now feasible. Accessing dose information from these sources is more rigorous and less prone to selection biases or judgements around 'standard' scanning practices the current DRL survey methodologies risk [31]. The barriers to participation in ongoing DRL surveys, protocol and dose audits are primarily motivational or cultural rather than technical. Modern CT and hospital information systems are capable of generating comprehensive databases on the scanning habits of providers and practice networks, rather than methodologically limited surveys prone to selective sampling, participation biases and low industry participation rates [32].

\section{Unnecessary use of CT}

Incentives within the Australian health system are different depending upon the setting. The MBS operates as a 
fee-for-service system, where MBS items include a description that determines the capacity for a given service's eligibility for payment to the provider of a set fee (schedule fee) from the federal government. Essentially, if a service does not fit the description of an MBS item there will be no subsidisation available from the federal government. Providers are able to charge the patient service fees in excess of the schedule fee; ultimately the difference is borne by the patient. Funding of public hospital services is via a separate mechanism negotiated as complex state and federal government health care funding agreements and is not based upon a fee-for-service model; leaving public hospital patients discharged without fee. Australian public hospitals are subject to more diffuse incentives regarding service delivery. The primary focus of recent health care funding agreements has been placing downward pressure on growing expenditure trends.

In order to restrict utilisation to indications shown to be cost-effective, the MBS has recently begun to incorporate specific clinical indications in the MBS item descriptors. This has been largely confined to newer technologies, such as Positron Emission Tomography (PET) and Magnetic Resonance Imaging (MRI). Narrowing the scope for use of CT is one possible mechanism to provide greater assurance of appropriate use. However, to re-write MBS item descriptions and establish clinical parameters for a scan to be performed, where none was included previously, requires substantial engagement with medical and imaging stakeholders.

Similar to other health systems, Australian GPs are able to refer patients for CT scans (performed overwhelmingly in MBS subsidised private services). There are concerns not all GPs appreciate the levels of radiation dose involved in a CT scan. Several studies have found specialists and GPs inaccurately estimate specific or relative CT doses in comparison to chest $\mathrm{x}$-rays [33-37]. A systematic review of physician surveys found CT dose underestimations in a very high proportion of physicians (60-99\%) [33]. The same systematic review noted attendance of a radiation protection course was positively associated with better knowledge of CT doses in two of five surveys that asked subjects about their education or experience [33].

Inaccurate perceptions among the group of doctors most accessible to the public and capable of referring patients to CT scans subsidised with unrestrictive MBS items runs the risk of inappropriate use. Several studies have found as much as $30 \%$ of all imaging tests are inappropriate in some fashion [38-40]. In some instances no imaging test is warranted at all, but more often are the situations where an alternative modality could have been utilised with lower or no radiation burden such as ultrasound, a plain radiograph or, MRI imaging. While the different modalities are not completely interchangeable, there is evidence use of these alternative services can offset some of the workload borne by CT machines and, by so doing, reduce the population radiation exposure burden and consequent cancer incidence [41]. Furthermore, previous publications have argued the economic cost of the radiation dose saved by using MRI rather than CT should be factored into cost-benefit assessments of MRI machines $[42,43]$.

Improved training of GPs could potentially reduce inappropriate CT scans and use of streamlined referral schema ('diagnostic imaging pathways') can assist the implementation of the ALARA principle [19]. More extreme measures such as removing GP referral for CT scanning would not only meet considerable political resistance, but would also be inconvenient to patients and increase demand for medical specialist services.

\section{Strengths and limitations}

The data collections used in this study were robust routinely collected administrative records of CT services used in both the public hospital and MBS settings. A significant strength of this study is the use of both public hospital and MBS data to capture the whole health care system rather than the limited examination of only MBS CT data in international comparisons, such as the OECD and UNSCEAR reports $[17,18]$. The ABS census data provide the best available population figures for the estimation of rates and relative risk. WA also provides a representative sample of the national population [44].

A limitation to the study was the lack of clinical information within the data sources beyond general demographic information and the standard descriptions associated with CT scan codes. This necessarily limits our capacity to comment on the trends observed, especially regarding systematic differences in clinical indication and patient medical history, to broader more systematic issues regarding economic incentives and radiation risk management regulation. Our study was also limited to adults and in doing so the MBS aggregate data for 15 to 24 year olds was cut by proportion to an 18 to 24 year old group; this assumes a consistent level of CT use for each year group in this age group. Paediatric radiology operates within a different clinical culture, especially within the hospital system, limiting the applicability of the results of this paper to children's CT services [3]. We are unable to provide an accurate comparison of WA with other states in terms of hospital CT services due to the diversity of state health system organisations. Each Australian state is also responsible for CT machine licencing with limited uniformity of reporting of machine numbers, making a comparison of CT machine prevalence between states or nationally impracticable.

\section{Conclusion}

Western Australian private practice CT scans constituted the bulk of CT services from mid-2006 to mid-2012. The 
public hospital sector $\mathrm{CT}$ scans were more likely to be performed on males; patients aged over 85 years and were predominantly head scans, whereas private practices tended to scan females and relatively younger patients with a diverse range of CT scan types, including those capturing anatomical sites with greater radiosensitivity. These two sectors present substantially different risk profiles with respect to CT utilisation. Private radiology providers, operating in the context of potentially contradictory commercial and radiation protection signals, perform the majority of CT scans with substantial subsidisation from the federal government. Appropriate levels of regulation, review and rationing of access are essential for any medical technology, especially with the potential for public health burden present in CT scanning. Australia has several avenues for expansion or refinement of acceptable dose levels, CT services eligible for funding, alternative diagnostic modalities and physician radiation dose training to ensure individual and population medical radiation dose is as low as reasonably achievable.

\section{Competing interests}

The authors declare that they have no competing interests.

\section{Authors' contributions}

DG performed the analyses, collaborated in study design, prepared manuscript drafts and revisions. RM conceived of study, collaborated in study design, assisted analyses and helped draft manuscript. DH collaborated in study design, helped draft manuscript. All authors have read and approved the final manuscript.

\section{Acknowledgements}

The authors would like to acknowledge the funding source for this research, the National Health and Medical Research Council (NHMRC). We would also like to acknowledge the data sources for this study; the Western Australian Department of Health, the Australian Government Medicare Benefits Schedule and the Australia Bureau of Statistics. The NHMRC and data sources did not have any role in the study design, data analysis, data interpretation or the decision to submit for publication.

\section{Author details}

${ }^{1}$ School of Population Health, University of Western Australia, Perth, Australia. ${ }^{2}$ Faculty of Health Sciences, Curtin University, Perth, Australia. ${ }^{3}$ Centre for Health Services Research, School of Population Health (M431), The University of Western Australia, 35 Stirling Hwy, Crawley, WA 6009, Australia.

Received: 12 November 2013 Accepted: 13 October 2014

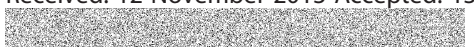

\section{References}

1. Commission E: European Guidelines on Quality Criteria for Computed Tomography. EUR 16262 EN. Luxenburg: Office for Official Publications of the European Communities; 1999

2. OECD StatExtracts: [http://stats.oecd.org]

3. Kalender WA, Wolf H, Suess C: Dose reduction in CT by anatomically adapted tube current modulation. Part II Phantom measurements. Med Phys 1999, 26:2248-2253. 26

4. Shrimpton PC, Wall BF: The increasing importance of $x$-ray computed tomography as a source of medical exposure. Radiat Prot Dosim 1995, 57:413-415

5. Wall BF: Implementation of DRLs in the UK. Radiat Prot Dosim 2005, 114(1-3):183-187.

6. Golding SJ, Shrimpton PC: Radiation Dose in CT: are we meeting the challenge? British J Radiol 2002, 75:1-4.
7. Moorin RE, Forsyth R, Gibson D, Fox R: Radiation dosimetry assessment of routine CT scanning protocols used in Western Australia. J Radiol Prot 2013, 33:295-312.

8. Pearce MS, Little MP, McHugh K, Lee C, Kim KP, Howe NL, Ronkers CM Rajaraman P, Craft AW, Parker L, and Berrington de Gonzalez A: Radiation exposure from CT scans in childhood and subsequent risk of leukaemia and brain tumours: a retrospective cohort study. Lancet 2012. Published online June 7 DOI: 10.1016/S0140-6736(12)60815-0.

9. Mathews JD, Forsythe AV, Brady Z, Butler MW, Goergen SK, Byrnes GB, Giles GG, Wallace AB, Anderson PR, Guiver TA, McGale P, Cain TM, Dowty JG, Bickerstaffe AC, Darby SC: Cancer risk in 680000 people exposed to computed tomography scans in childhood or adolescence: data linkage study of 11 million Australians. Br Med J 2013, 346:f2360

10. Sodickson A: CT radiation risks coming into clearer focus. Br Med J 2013, 346:f3102.

11. Berrington De Gonzalez A, Darby S: Risk of cancer from diagnostic x-rays: estimates for the UK and 14 other countries. Lancet 2004, 363:345-351.

12. Berrington De Gonzalez A, Mahesh M, Kim KP, Bhargavan M, Lewis R, Mettler F, Land C: Projected cancer risks from computed tomographic scans performed in the united states in 2007. Arch Intern Med 2009, 169(22):2071-2077.

13. Redberg RF: Cancer risks and radiation exposure from computed tomographic scans. Arch Intern Med 2009, 169(22):2049-2050

14. Brenner DJ, Hall EJ: Computed Tomography: An increasing source of radiation exposure. N Engl J Med 2007, 357:227-2284.

15. Mettler FAJ, Wiest PW, Locken JA, Kelsey CA: CT scanning: patterns of use and dose. J Radiol Prot 2000, 20(4):353-359.

16. Zweifel P, Ferrari M: Is there a Sisyphus Syndrome in health care? In Health Economics Worldwide. Edited by Zweifel P, Frech H III. Dordrecht, NL: Kluwer; 1992

17. United Nations Scientific Committee on the Effects of Atomic Radiation: Sources and Effects of lonizing Radiation. New York: United Nations; 2000.

18. OECD: Health at a glance 2011: OECD Indicators. OECD Publishing. http:// dx.doi.org/10.1787/health_glance-2011-en; 2011

19. Diagnostic Imaging Pathways. [http//wwww.magingpathways.health.wa.gov.au/]

20. Committee to Assess Health Risks from Exposure to Low Levels of lonizing Radiation: Health risks from exposure to low levels of ionizing radiation: BEIR VII-Phase 2. In.: Board on Radiation Effects Research, Division on Earth and Life Studies, National Research Council.; 2006

21. Medicare Item Reports. [https://www.medicareaustralia.gov.au/statistics/ mbs_item.shtml]

22. Australian Bureau of Statistics: 3101.0 - Australian Demographic Statistics, Dec 2011. Estimated Resident Population by Single Year of age 1971-2011 in Western Australia. Canberra: ABS; 2012.

23. Strauss KJ, Kaste SC: The ALARA (as Low as reasonably achievable) concept in pediatric interventional and fluoroscopic imaging: striving to keep radiation doses as Low as possible during fluoroscopy of pediatric patients-a white paper executive summary. Radiology 2006, 240(3):621-622

24. Mayo JR, Aldrich J, Müller NL: Radiation exposure at chest CT: a statement of the fleischner Society1. Radiology 2003, 228(1):15-21.

25. Gibson DA, Moorin RE, Semmens J, Holman DAJ: The disproportionate risk burden of $\mathrm{CT}$ scanning on females and younger adults in Australia: a retrospective cohort study. Aust N Z J Public Health 2014, 38(5):441-448

26. International Commission on Radiological Protection: Managing patient dose in computed tomography. International Commission on Radiological Protection Publication 87. Ann ICRP 2000, 30:4.

27. International Commission on Radiological Protection: Recomendations of the International Commission on Radiological Protection publication number 103. In.: ICRP; 2007

28. Commission E, Dose Datamed Project: European Guidance on Estimating Population Doses from Medical X-Ray Procedures, Radiation Protection No.154. Chilton, Didcot, Oxfordshire: Health Protection Agency; 2008.

29. Current Australian National Diagnostic Reference Levels for MDCT [http://www.arpansa.gov.au/services/NDRL/current.cfm]

30. Shrimpton PC, Hillier MC, Lewis MA, Dunn M: Doses from computed tomography (CT) examinations in the UK - NRPB-W67. Oxon: National Radiological Protection Board; 2003

31. Takx RA, Schoepf UJ, Moscariello A, Das M, Rowe G, Schoenberg SO, Fink C, Henzler T: Coronary CT angiography: Comparison of a novel iterative 
reconstruction with filtered back projection for reconstruction of low-dose CT-Initial experience. Eur J Radio/ 2013, 82(2):275-280.

32. Shrimpton PC, Jones DG, Hillier MC, Wall BF, Le Heron JC, Faulkner K: Survey of CT Practice in the UK. Part 2: Dosimetric Aspects. Chilton: NRPB-R249; 1991.

33. Krille L, Hammer GP, Merzenich H, Zeeb H: Systematic review on physician's knowledge about radiation doses and radiation risks of computed tomography. Eur J Radiol 2010, 76(1):36-41.

34. Lee $\mathrm{Cl}$, Haims AH, Monico EP, Brink JA, Forman HP: Diagnostic CT scans: Assessment of patient, physician, and radiologist awareness of radiation dose and possible risks. Radiology 2004, 231(2):393-398.

35. Perko T: Radiation risk perception: a discrepancy between the experts and the general population. J Environ Radioact 2014, 133:86-91.

36. Zhou G, Wong D, Nguyen L, Mendelson R: Student and intern awareness of ionising radiation exposure from common diagnostic imaging procedures. J Med Imaging Radiat Oncol 2010, 54(1):17-23.

37. Lee R, Chu W, Graham C, Rainer T, Ahuja A: Knowledge of radiation exposure in common radiological investigations: a comparison between radiologists and non-radiologists. Emerg Med J 2012, 29:306-308.

38. Bairstow P, Persaud J, Mendelson R, Long N: Reducing inappropriate diagnostic practice through education and decision support. Int I Qual Health Care 2010, 22(3):194-200.

39. Simpson G, Hartrick G: Use of thoracic CT by general practitioners. Med J Aus 2007, 187:43-46.

40. Lehnert B, Bree R: Analysis of the appropriateness of outpatient CT and MRI referred from primary care clinics at an academic medical center: how critical is the need for improved decision support? J Amer Coll Radiol 2010, 7(3):192-197.

41. Clarke JC, Cranley K, Kelly BE, Bell K, Smith PHS: Provision of MRI can significantly reduce CT collective dose. British J Radiol 2001, 74:926-931.

42. DHSS (NI): Report of a CT radiation dose survey in Northern Ireland. Edited by Party CISAC-CTW; June 1998

43. Clarke J, Cranley K, Robinson J, Smith P, Workman A: Application of draft European Commission reference levels to a regional CT dose survey. Br J Radiol 2000, 73:43-50.

44. Winklehner A, Karlo C, Puippe G, Schmidt B, Flohr T, Goetti R, Pfammatter T, Frauenfelder $\mathrm{T}$, Alkadhi $\mathrm{H}$ : Raw data-based iterative reconstruction in body CTA: evaluation of radiation dose saving potential. Eur Radiol 2011, 21(12):2521-2526.

doi:10.1186/s12913-014-0526-0

Cite this article as: Gibson et al:: Cohort study of Western Australia computed tomography utilisation patterns and their policy implications. BMC Health Services Research 2014 14:526.

\section{Submit your next manuscript to BioMed Central and take full advantage of:}

- Convenient online submission

- Thorough peer review

- No space constraints or color figure charges

- Immediate publication on acceptance

- Inclusion in PubMed, CAS, Scopus and Google Scholar

- Research which is freely available for redistribution

Submit your manuscript at www.biomedcentral.com/submit
Ciomed Central 